\section{Kommunikation Neuer Technologien. Das Beispiel Biotechnologie}

\author{
von Marc-Denis Weitze, Deutsche Akade- \\ mie der Technikwissenschaften (acatech), \\ München, Armin Grunwald, Institut für Tech- \\ nikfolgenabschätzung und Systemanalyse \\ (ITAS), Karlsruhe, Alfred Pühler, Centrum für \\ Biotechnologie an der Universität Bielefeld, \\ und Wolfgang $M$. Heckl, TU München und \\ Deutsches Museum
}

Biotechnologie ist ein exemplarischer Fall „Neuer Technologien“, die die Möglichkeitshorizonte moderner Gesellschaften definieren und deren Diskussion Einblick in zentrale gesellschaftliche Interessenlagen, Konfliktlinien und Entwicklungsdynamiken erlauben. Eine sozialwissenschaftliche und historische Perspektive auf die Kommunikation, Debatten und Kontroversen um Biotechnologie gibt Hinweise auf neue, mehr Erfolg versprechende Kommunikationsansätze. Visionäre Forschungsfelder wie die Künstliche Fotosynthese und kontroverse Bereiche wie die sog. Genom-Chirurgie stellen immer wieder von neuem die Frage, wie neben Vertretern von Wissenschaft und Wirtschaft auch Vertreter von (Teil-)Öffentlichkeit(en) ihre Ideen, Erwartungen und Befürchtungen einbringen können und wie sich Öffentlichkeit frühzeitig in Innovationsprozesse einbeziehen lässt.

Biotechnology, or the application of science and technology to living organisms, is a prime example of the "new technologies" that define the extent of opportunities currently available to our modern society. Sociologically and historically analyzing communication activities, debates and controversies can inform us about novel and promosing communication approaches. Visionary fields of research such as Artificial Photosynthesis as well as controversial fields such as Genome Editing bring about the question how the use and the development of new technologies can be shaped as part of a comprehensive process of developing consensus on scientific requirements, interests, values and preferences, taking into account all interested groups of society.

\section{Perspektiven der Biotechnologie-} Kommunikation

Seit vier Jahrzehnten wird BiotechnologieKommunikation betrieben. Gerade in den vergangenen Jahren entstanden zu diesem Thema in Deutschland immer neue Formate, die dem Dialog zwischen Wissenschaft und Gesellschaft dienen sollen - neben Informationsangeboten auf Papier, im Fernsehen und Internet sind dies beispielsweise Dialogveranstaltungen wie Bürgerkonferenzen oder Ausstellungen mit Besucherlaboren. Trotz allem ist Akzeptanz etwa für Grüne Gentechnik in Deutschland bis heute nicht gegeben, so dass Unternehmen wie die BASF die Entwicklungsarbeit hierzulande als perspektivenlos einschätzen und ihre Aktivitäten in die USA verlagert haben.

Um an einem Beispiel die Herausforderungen der Technikkommunikation detailliert auf der Basis vieler Erfahrungen zu betrachten, hat acatech im Rahmen eines zweijährigen Projekts „Kommunikation Neuer Technologien Biotechnologie“ (2010-2012) Erfahrungen aus Kontroversen und Kommunikationsmaßnahmen zur Grünen Gentechnik und anderen Feldern der Biotechnologie in kommunikations- und sozialwissenschaftlicher sowie historischer Perspektive analysiert. Die Projektgruppe hat hierzu Expertisen eingeholt (Weitze et al. 2012), Workshops zur Diskussion der Zwischenergebnisse veranstaltet und den Dialog mit Multiplikatoren wie Journalisten genauso gesucht wie mit Umweltverbänden. Die Projektergebnisse und Empfehlungen (acatech 2012a) werden im Weiteren dargestellt.

In der Wissenschafts- und Technikkommunikation, so die wesentliche Erkenntnis für die Akademie, gibt es keine Patentrezepte: Kommunikation und Beteiligung sind immer an die jeweilige Einzigartigkeit der Themen und der Situation auszurichten. Grundsätzlich lässt sich festhalten, dass Kontroversen dabei wichtig sind und sich durch Kommunikation allein nicht aus der Welt schaffen lassen. Schließlich sind Kontroversen ein wesentliches Element sowohl des wissenschaftlichen Erkenntnisgewinns als auch der Wissenschaftskommunikation. Öffentliche Kontroversen von vorneherein als ,mangelnde Akzeptanz" der Wissenschaft oder der Technik zu deuten, greift zu kurz. 
Erfahrungen aus der Vergangenheit zeigen des Weiteren, dass es vergeblich ist, bestehende Einstellungen in der Bevölkerung direkt beeinflussen zu wollen. Vielmehr müssen diese, ebenso wie Spezifika der Rezeption, zunächst als Randbedingungen bei der Gestaltung von Kommunikation berücksichtigt werden. Umgekehrt gibt es auch auf Seiten der Wissenschaftler besonders stabile (Fehl-)Vorstellungen. So meinen viele Wissenschaftler, dass Vorteile Neuer Technologien aufgrund mangelnder Information von ,ignoranten Laien" übersehen würden - wobei es häufig gerade Vertreter der gebildeteren Schichten sind, die Bedenken gegenüber den Risiken Neuer Technologien anmelden (z. B. Kahan et al. 2009).

Die Empfehlungen der Akademien richten sich an Wissenschaft, Wirtschaft, Politik, Hochschulen und Wissenschaftsforschung (s. Kasten 1).

\section{2 „Clearingstelle“ Biotechnologie}

In Empfehlung 7 wird die Einrichtung einer "Clearingstelle“ vorgeschlagen, die Informationen zu kontroversen Themen aufbereitet. Angesichts der Informationsflut und der Vielzahl an Interessenvertretern kann solch eine Stelle nicht dazu dienen, die ,richtige“ Sichtweise des Problems darzustellen, aber die Pluralität sichtbar zu machen, um eine verständigungsorientierte Basis für einen konstruktiven Dialog zu schaffen. Ähnliche Ansätze wurden bereits von verschiedenen Akteuren gedacht: So forderte die Hightech-Strategie der Bunderegierung: „Ob real oder virtuell - alle Bürgerinnen und Bürger sollen leichten Zugang zu Diskussionsforen und vorhandenen Informationen haben." (BMBF 2014, S. 45) Der Beirat für Wissenschaft und Technik des Präsi-

\section{Kasten 1: acatech-Empfehlungen zur Biotechnologie-Kommunikation}

\section{Empfehlungen an Wissenschaft und Wirtschaft}

1. acatech empfiehlt, die Quellen von Information transparent zu machen und Ziele der Kommunikation zu reflektieren.

2. acatech empfiehlt zu berücksichtigen, um welche (Teil-)Themen es in der Kommunikation jeweils geht.

3. acatech empfiehlt, nicht nur die Inhalte der Wissenschaft, sondern auch die Prozesse des Erkenntnisgewinns in den entsprechenden Wissenschaftszweigen, die Methoden der Risiko- und Chancenabschätzung und die Verfahren der politischen Regulation gemeinsam mit den Ergebnissen zu kommunizieren.

4. acatech empfiehlt, dass sich Wissenschaftler, die als Kommunikatoren schon heute beträchtliche finanzielle und zeitliche Ressourcen investieren, um mehr Sichtbarkeit bemühen und ihre Glaubwürdigkeit pflegen gegenüber teilweise weniger sachkundigen, aber medial viel stärker präsenten Meinungsführern.

5. acatech empfiehlt im Sinne einer Zielgruppenorientierung einen problem- statt technologieorientierten Zugang in der Kommunikation mit der Öffentlichkeit.

\section{Empfehlungen an Wissenschaft, Wirtschaft und Politik}

6. acatech empfiehlt, die Positionen und Bewertungen der einzelnen Stakeholder, also auch jener außerhalb der Wissenschaft, in allen Kommunikationsprozessen mit Respekt zu betrachten, unvoreingenommen zu reflektieren und ernst zu nehmen. Hinsichtlich des Dialogs zwischen Wissenschaft und Gesellschaft ist zu untersuchen, wie Informationen und Meinungen von Seiten der Öffentlichkeit systematisch in Wissenschaft und Wirtschaft wahrgenommen bzw. aufgenommen werden können.

7. acatech empfiehlt die Einrichtung einer „Clearingstelle“ im Internet, die Informationen zu kontroversen Themen unabhängig von allen Interessengruppen und ausgewogen aufbereitet.

8. acatech empfiehlt, zusätzlich zu den bewährten Modellen der Kommunikation und der Beteiligung neue, innovative Formen, vor allem im Zusammenhang mit den neuen Medien (Web 2.0) weiter zu erproben, zu entwickeln und zu evaluieren.

\section{Empfehlung an Hochschulen}

9. acatech empfiehlt, Grundfertigkeiten für eine adressatengerechte und sachlich fundierte Wissenschaftskommunikation in der Ausbildung zu verankern.

Empfehlung an Wissenschaftsforschung

10. acatech empfiehlt, wissenschaftliche Studien zu initiieren, die systematisch theoretische Erkenntnisse mit praktischen Erfahrungen zusammen führen.

Quelle: acatech 2012a, S. 36-38 
denten der Europäischen Kommission hat empfohlen, dass die europäische Kommission eine Art „Radarsystem“ aufbaut, mit dem eine frühzeitige Analyse der Chancen und Risiken ermöglicht werden soll, und in das auch Meinungen der Bürger einfließen sollen. (EC 2013, S. 16) Und Uwe Schneidewind formuliert: „Das Zusammenwirken staatlicher und privater Akteure muss so geändert werden, dass Ungleichgewichte aufgehoben, die Fixierung auf technologische und ökonomische literacy gelöst und stattdessen Orte der Wissensintegration geschaffen werden." (Schneidewind 2013)

Auf einem Workshop (Berlin, 31.10.2014) hat acatech mit Akteuren aus Wissenschaft, Wirtschaft und Zivilgesellschaft diskutiert, welche konkreten Ziele mit solch einer Plattform verfolgt werden könnten - z. B. Politikberatung, Gesellschaftsberatung, wissenschaftspolitische Partizipation, Akzeptanzbeschaffung oder ein Radarsystem. Wie wäre die Zielgruppe genauer zu umreiBen (Bürger, Wirtschaft, Wissenschaft, Medien, Kritiker, Verbände, öffentliche Stellen,...)?

Einzelne Beispiele zeigen die Bandbreite an bereits existierenden Formaten zur Biotechnologie, die in Richtung einer Clearingstelle weisen:

- Das Webportal „Pflanzen.Forschung.Ethik.“2 des Instituts Technik-Theologie-Naturwissenschaften an der Ludwig-MaximiliansUniversität München ,informiert anhand verständlicher Texte, übersichtlicher Grafiken und kurzer Videos über das weite Themenfeld der modernen Pflanzenforschung. Das Portal diskutiert soziale, ökonomische und ökologische Fragen und fängt Stimmungen und Meinungen von Akteuren, nicht zuletzt mit Fokus auf Bayern, ein." Auf dieser Internetseite liegt der Fokus auf Grüner Biotechnologie mit vielen Beispielen und Experteninterviews. Darüber hinaus gibt es eine Rubrik „Werte und Moral“. Jeder Besucher der Internetseite hat die Möglichkeit, diverse Fallbeispiele zu begutachten und sich somit zu beteiligen.

- Das Online-Portal biotechnologie.de ${ }^{3}$, das die BIOCOM AG im Auftrag des Bundesministeriums für Bildung und Forschung seit 2005 betreibt, versteht sich selbst als „die führende One-Stop-Adresse für alle Fragen rund um die Biotechnologie und Life Sciences". Hier ist eine Mischung aus Grundwissen und aktuellen Forschungsergebnissen zur Biotechnologie zu finden. Außerdem ist eine Rubrik mit Informationen für Schüler, Studierende und Lehrer zu finden.

- Der „Informationsdienst Gentechnik ${ }^{\text {“4 }}$ ist eine Seite der Zukunftsstiftung Landwirtschaft: Zur Unterstützung derjenigen, die sich in Stadt und Land für die Sicherung der gentechnikfreien Landwirtschaft und Ernährung engagieren, haben Umwelt-, Wirtschafts-, Verbraucher und Bauernverbände gemeinsam den Informationsdienst Gentechnik ins Leben gerufen.

Einige Beispiele aus Großbritannien:

- Sense about Science ${ }^{5}$ versteht sich als ,an independent trust with a small office team, run by a board of trustees, assisted by a 40 -strong advisory council“. Die Finanzierung erfolgt auf breiter Basis durch u. a. Stiftungen, Wissenschaftsorganisationen, Verlage oder Einzelpersonen. Diese Seite möchte den Nutzern ermöglichen, aktuelle Forschungsergebnisse und Diskussionen einzuordnen, zu bewerten, sich einzubringen (s. auch askforevidence.org).

- Das Science Media Center ${ }^{6}$ „has its roots in the influential House of Lords Science and Technology Select Committee third report on Science and Society, which wanted to renew public trust in science. [...] The mission is to provide, for the benefit of the public and policymakers, accurate and evidence-based information about science and engineering through the media, particularly on controversial and headline news stories when most confusion and misinformation occurs." Die Finanzierung erfolgt auf breiter Basis, u. a. durch Unternehmen, Stiftungen, Wissenschaftsorganisationen. Es richtet sich hauptsächlich an Journalisten, Wissenschaftler und Presseagenturen. Dabei gibt es auch Formate, die gezielt Wissenschaftler und Journalisten zusammen bringen. ${ }^{7}$

Die Diskussion im Rahmen des Berliner Workshops hatte weitere Fragen aufgeworfen. So wäre zu klären, inwieweit solche Plattformen tatsächlich den wissenschaftspolitischen Diskurs in der Demokratie fördern und nicht der bloßen PR bzw. Akzeptanzbeschaffung für gesetzte Technologien dienen oder der Meinungshomogenisierung 
zu solchen Fragen. Wie gelingt es, Biotechnologie in gesellschaftliche Kontexte zu stellen und zu verstehen, wie verschiedene Positionen dazu entstehen bzw. sichtbar werden? Wer übernimmt welche Rollen? Sind Wissenschaftler neutrale Instanz oder Meinungsträger? Sollen Journalisten die Rolle eines Moderators übernehmen?

Die Ausgestaltung würde vielfältige Variablen betreffen: Wenn es ein Ziel wäre, Fakten zu sammeln und zu sichten, anschließend zu arrangieren, um eine gemeinsame Basis für einen Dialog zu schaffen, vorhandene Argumente aufzuarbeiten, einzuordnen und für einen Dialog zu strukturieren - wie gelingen die Auswahl der Fakten und die Strukturierung der Argumente im Konsens mit den Beteiligten? Wer betreibt dann die Plattform (z. B. private Initiativen/Stiftungen, Universitäten, Netzwerkinitiativen, NGOs, Wissenschaftsjournalisten, Agenturen, ...)? Wer finanziert die Plattform (z. B. Ministerien, Stiftungen, Unternehmen, ...)? Welche Inhalte sollen dargestellt werden (z. B. Forschung, gesellschaftliche Kontroversen, neu aufkommende Themen, Darstellung der Fakten, Argumente und des Diskurses, Verbraucherrelevanz, gesetzliche Regulierung,...)? Wie interaktiv/partizipativ soll die Internetplattform gestaltet sein? Welche Formate sind in der Plattform enthalten? Was sind die Erfolgskriterien (z. B. Zielerreichung, Nutzungsintensität, relative Nutzung der einzelnen Inhalte, Nachfrage der Interaktivität, neue Aspekte im Dialog, ...)? Wie soll die Dialogplattform in der Öffentlichkeit publik gemacht werden? Wäre der Begriff „Clearingstelle“ überhaupt angemessen, oder klingt das nach Kontrollinstanz, die entscheidet, welche Informationen an die Öffentlichkeit gelangen darf und welche nicht? acatech wird der Idee einer „Clearingstelle“ und den damit verbundenen Fragen in Zukunft weiter nachgehen.

\section{Vom Defizit zum Dialog}

Empfehlung 6 zur Biotechnologie-Kommunikation betrifft die Herausforderung, Akteure und Stimmen jenseits von Wissenschaft und Wirtschaft in den Dialog zu Neuen Technologien einzubeziehen. Sie knüpft an Forderungen der Wissenschafts- und Forschungspolitik an, wie sie etwa in einem vom BUND herausgegebenen „Plädoyer für eine Wis- senschaft für und mit der Gesellschaft" formuliert worden sind: „Mit welchen Fragestellungen sich Wissenschaft beschäftigt, darf [...] nicht alleine durch einzelne gesellschaftliche Gruppen und durch ökonomische Interessen bestimmt sein. Forschungsfelder und -themen müssen möglichst pluralistisch mit der Wissenschaft festgelegt werden. Es muss insbesondere transparent sein, wer auf die Definition von Forschungsthemen Einfluss nimmt“" (BUND 2012, S. 5) und: „Gesellschaftliche Gruppen sind [...] schon viel früher in die Prozesse zur Definition von Forschungsprogrammen einzubeziehen" (BUND 2012, S. 11).

Tatsächlich setzte Kommunikation in der Vergangenheit häufig erst ein, nachdem eine Technologie entwickelt war und Meinungen dazu bereits verfestigt waren. Hochglanzbroschüren sollten Bewunderung hervorrufen und für $\mathrm{Zu}$ stimmung sorgen. Lange Zeit versuchte man, dieses Verhältnis von Wissenschaft und Öffentlichkeit mit dem Defizitmodell zu beschreiben: Die Wissenschaft definiert den Stand des Wissens. Dieses Wissen wird vereinfacht an die Öffentlichkeit weitergegeben. Die bleibt passiv und soll die Neue Technologie allenfalls akzeptieren. Seit den 1990er Jahren weiß man jedoch, nicht zuletzt nach der Diskussion um Kernenergie und Grüne Gentechnik: Mehr (popularisiertes) Wissen führt keineswegs automatisch zu mehr Akzeptanz. Der Trend geht hin zum Dialog - insbesondere wenn es um Themen geht, die mit Unsicherheiten und Risiken behaftet sind und die Öffentlichkeit direkt betreffen. Dialog bedeutet Verständigung in beide Richtungen, ermöglicht den Austausch von Meinungen und Sichtweisen und damit eine sachgerechte und ausgewogene Kommunikation.

Die Argumente für „mehr Dialog“ reichen von demokratischer Legitimation über eine Erweiterung der Wissensbasis bis hin zu Fragen der Akzeptanz (acatech 2011):

- Relevantes Wissen findet sich auch außerhalb der Wissenschaft. Grenzen des Fachwissens wurden unter anderem in biomedizinischer Forschung, in der Agrarpolitik und in Umweltdebatten deutlich. Angesichts immer weiter voran schreitender gesellschaftlicher Differenzierung und der Auflösung gemeinsamer Erfahrungswelten, tut es Not, die verschiedenen Wissensformen (aus Wissenschaft, Wirtschaft und Ge- 
sellschaft) zusammen zu bringen, zumal sich die Wissensproduktion und die Entwicklung neuer Technologien ständig beschleunigen.

- Entscheidungen nicht nur auf der Basis technischer Expertise, sondern auf derart verbreiteter Basis sind inhaltlich besser nachvollziehbar und „sozial robust" - eben weil es durch gesellschaftliches Wissen infiltriert und verbessert wurde und auf einem umfassenderen Spektrum von Perspektiven und Techniken basiert (Nowotny et al. 2001, S. 166ff.).

- Angesichts des immer stärkeren Einflusses Neuer Technologien in der Gesellschaft sind neben dem Fachwissen der Experten Wertvorstellungen, Zukunftsvisionen und Wünsche der Bürger relevant.

- Zudem ist eine grundsätzliche Aufgeschlossenheit gegenüber technischen Innovationen, die etwa durch Dialogformate gestärkt werden kann, heute ein wirtschaftlicher Faktor.

\section{Künstliche Fotosynthese gemeinsam gestalten - ein Modellexperiment}

Im Projekt „Künstliche Fotosynthese - Entwicklung von Technikzukünften" von acatech (20132015) wurde eruiert, wie die Öffentlichkeit (und damit Nutzer und Betroffene) in die Entwicklung von Technikzukünften (acatech 2012b) einbezogen werden kann. Statt Innovation separat von der Öffentlichkeit entstehen zu lassen und nachträglich für die Diffusion zu sorgen sowie Folgen zu regulieren und Akzeptanz zu schaffen, sollen hier bereits in der Entwicklungsphase (und nicht erst in der anschließenden Nutzungsphase) Entwickler, Regulatoren, potenzielle Nutzer und die allgemeine Öffentlichkeit einbezogen werden (acatech 2016).

Als Innovationsfeld wurde die Künstliche Fotosynthese gewählt: Vor dem Hintergrund eines wachsenden globalen Energiebedarfs, schwindender fossiler Energieträger und dem Wunsch, die $\mathrm{CO}_{2}$-Emissionen $\mathrm{zu}$ reduzieren, ist Künstliche Fotosynthese eine visionäre Technologie, die zum Energiemix einen wichtigen Beitrag leisten könnte. Nach dem Vorbild der Pflanzen nutzt die Künstliche Fotosynthese Sonnenlicht, um aus den Rohstoffen Wasser und $\mathrm{CO}_{2}$ energiereiche Verbin-

\section{Kasten 2: Das Gläserne Forscherlabor}

Eine Dialogmöglichkeit, die neben einem Austausch zudem den Prozess der Forschung stärker in der Wissenschaftskommunikation sichtbar macht, ist das Gläserne Forscherlabor (http://www.openlab.edu.tum.de). Im Unterschied zu den Schüler- und Besucherlaboren, in denen unter Anleitung komplexe Experimente etwa im Bereich Genetik durchgeführt werden können, lassen sich hier die Wissenschaftler während ihrer Arbeit über die Schulter schauen und befragen. Eine Handvoll Doktoranden betreibt das Labor im Deutschen Museum, das statt im Keller einer Forschungseinrichtung (oder im sprichwörtlichen „Elfenbeinturm“) nun in die öffentliche Arena eines Wissenschaftsmuseums gezogen ist.

Demonstrationsexperimente, Präsentationsmonitore und Schautafeln ergänzen das Labor, das ansonsten ausgerüstet ist wie ein Universitätslabor: Probenpräparation, Messen und Auswerten sind hier in der gleichen Qualität möglich, so dass hier tatsächlich „richtige“ Forschung stattfindet.

Die Doktoranden widmen sich also neben ihrer Forschung dem Dialog mit der Öffentlichkeit. Dazu müssen sie einerseits alle Abläufe und Techniken nicht nur selbst verstanden haben, sondern auch verständlich anderen erläutern können. Andererseits werden sie im Dialog mit Besuchern mit generellen Fragen zur Bio- und Nanotechnologie konfrontiert, etwa zu den möglichen Umweltauswirkungen. Und die Besucher erfahren sowohl die Faszination als auch die täglichen Mühen der Forschung. Sie zeigen sich insbesondere interessiert an der Motivation der Doktoranden, Forschung in diesem Feld zu betreiben.

dungen herzustellen - oder elektrische Energie, die entsprechend weiter umgewandelt werden kann.

So groß das Potenzial der Nutzung der Sonnenenergie ist, so groß sind bis heute aber die damit verbundenen technisch-wissenschaftlichen Fragestellungen. Sowohl hinsichtlich der Fotovoltaik, die als besonders teure regenerative Energie gilt, als auch hinsichtlich der künstlichen Fotosynthese werden derzeit verschiedene biobasierte Ansätze verfolgt. So könnte der Wirkungsgrad des natürlichen fotosynthetischen Systems erhöht werden, etwa mittels Gentechnik oder Synthetischer Biologie. Als ergänzend können Ansätze der lichtabhängigen Metallkatalyse betrachtet werden, bei denen Kohlendioxid etwa durch Fotokatalyse zu Methanol reduziert wird. Das Sonnenlicht soll dabei von organischen Farbstoffen absorbiert und seine Energie zur Aktivierung von $\mathrm{CO}_{2}$ verwendet werden. Hinsichtlich der technischen Nutzung der Fotosynthese liegt der Zeithorizont von marktfähigen Produkten bei 2050.

Dass Zukunftsvorstellungen eine entscheidende Rolle in gesellschaftlichen Technikdebat- 


\begin{abstract}
Kasten 3: Storytelling - Live und in Videos
von Wolfgang Chr. Goede, Wissenschaftsjournalist und Vorstandsmitglied (Sekretär) des Weltverbandes der Wissenschaftsjournalisten WFSJ

Seit Jahren leite ich Workshops über Storytelling. Während kleine Kinder und alte Menschen Geschichtenerzählen noch beherrschen, tut sich der demografische Mittelbau schwer damit. Die Durchakademisierung unserer Erziehung schult abstraktes Denken, nicht darstellerisches. Aber: Wenn knisterndes Lagerfeuer auf einem Monitor mitläuft, tun sich Storyteller leichter.

Der empirische Beweis für eine Hypothese der Kulturanthropologen: Am Lagerfeuer der Steinzeit lernte der frühe Homo sapiens Geselligkeit, Sprache; wurde er zum Erfinder. Beim Erzählen von Jagdberichten wurden Waffen und Strategien bewertet. Das lud auf der freien Wildbahn zu Experimenten ein. Dies war die Wiege unserer technischen Zivilisation - und von Hollywood.

Jeder spannende Film basiert auf Steinzeitfeuergeschichten. Beide leben von Helden, meist in Gegnerschaft verbunden, von Dramaturgie, zwischen Erfolg und Misserfolg, Liebe und Gewalt, großen Emotionen, guten und bösen Enden sowie Lehren daraus. Solche Heldenreisen finden wir in den großen Zeugnissen unserer Kultur, der Bibel und Nibelungensaga, Grimms Märchen und Harry Potter. Jeder Roman, jede Animation folgt dieser archaischen Rezeptur.

Für Forschungsergebnisse, die einem breiten Publikum plausibel werden sollen, heißt das: Sie müssen in dieses Steinzeitprogramm des modernen Homo sapiens transformiert werden.

Das acatech-Projekt „Technik gemeinsam gestalten“, bei dem Laien in den frühzeitigen Dialog mit Wissenschaftlern über die Chancen der Künstlichen Fotosynthese einbezogen wurden, machte sich Storytelling zunutze. Ich wurde beauftragt, zu drei Technikzukünften Geschichten zu finden. Die Narrative „Algenblüte“, „Nanozauber“, „Solarguerilla“ kleideten diverse Technologien in Hightech-Krimis:

Die Odyssee, aus mediterranen Braunalgen Ethanol zu gewinnen; ein von der Kern-Lobby verfolgter Nerd, der das Treibhausgas $\mathrm{CO}_{2}$ mit Nano-Kügelchen zu Methan veredelt; wie eine Plattenbausiedlung sich mit Solarfolien in einer Nacht-und-NebelAktion in ein lukratives Kraftwerk verwandeln will. Dass die Erzählungen beim Publikum zündeten, selbst bei 40 Grad Hitze, spricht für die Storytelling-Live-Strategie bei komplexen Forschungsthemen.

Dann der nächste Schritt: die Storys zum Erreichen größerer Menschenmengen in dreiminütige Videos umzuarbeiten. Reduktion der Handlungen auf Mono- und Dialoge; Stimme, Mimik und Posen aufeinander abstimmen; Requisiten und Kulissen (alle im Deutschen Museum) festlegen; Kameraperspektiven finden; fachgerechtes Schneiden der Filmsequenzen.

Videos haben Konjunktur. Handys verlangen nach bewegten Bildern. Kürze und Action sind gefragt. BBC-Clips sind nur noch 40 Sekunden lang. Das stellt riesige Herausforderungen an Qualität und Kreativität. Es war ein langer Weg vom Lagerfeuer der Steinzeit zum digitalen Lagerfeuer. Dieses eröffnet der Forschung nun neue, bisher wenig beschrittene Kommunikationswege.
\end{abstract}

ten spielen, ist etwa im Bereich der Nanotechnologie zu erkennen. Dabei werden Fragen wie die folgenden thematisiert: Wo liegen die Herausforderungen? Welche Technologien wollen die Nutzer, welche wichtige Gruppen der Gesellschaft und welche die Bürger? Wie schätzen diese Chancen und Risiken verschiedener Problemlösungen ein? Im Unterschied etwa zu Kernenergie und Grüner Gentechnik handelt es sich bei der Künstlichen Fotosynthese um ein durch Kontroversen bzw. verfestigte Meinungsbilder noch nicht „,vorbelastetes“ Feld. So scheint das kontroverse Potenzial dieser Technologie auf den ersten Blick eher gering. Jedoch können hier durch den möglichen Einsatz von Gentechnik oder Schwermetall-Katalysatoren auch durchaus kontroverse umweltrelevante und ethische Fragen erwachsen. Die wissenschaftlichen Ausgangspunkte und technischen Realisierungsmöglichkeiten sind bislang allenfalls in Ansätzen erkennbar.

Um die Künstliche Fotosynthese in ihrem frühen Forschungsstadium für interessierte Bürger verständlich zu machen, entwarf die Projektgruppe unterschiedliche Technikzukünfte. Diese
Methode der Technikkommunikation übersetzt Forschungsergebnisse in Geschichten, die beschreiben, wie eine mögliche Zukunft mit Künstlicher Fotosynthese aussehen kann. Sie beschreiben sowohl die mögliche zukünftige Gesellschaft als auch die Technik und können unterschiedlicher Gestalt sein, zum Beispiel wissenschaftliche Vorausschauen, literarische oder filmische Science Fiction-Szenarien. Die Technikzukünfte zur Künstlichen Fotosynthese, die die Projektgruppe für den Dialog mit der Öffentlichkeit entwarf, drehen sich um Mikroalgen und Wasserlinsen, die als grüne Zellfabriken energiereiche Stoffe produzieren; oder es drehte sich um Nanokügelchen, die in einem elektrokatalytischem Prozess aus Wasser und $\mathrm{CO}_{2}$-haltigen Industrieabgasen energiereiches Methangas herstellen. Eine andere beschreibt transparente organische Solarzellen, die als Baumaterialien aus Gebäudefassaden ein Kraftwerk zur Stromproduktion machen. Ein Wissenschaftsjournalist hat die noch immer stark in Wissenschaft und Technik verhafteten Technikzukünfte weitergesponnen in Geschichten (http://www.acatech.de/fotosynthese-geschichten und Kasten 3). 


\begin{abstract}
Kasten 4: Das Science Café als Dialog- und Beteiligungsformat von Rüdiger Goldschmidt, Zentrum für Interdisziplinäre Risiko- und Innovationsforschung (ZIRIUS), Universität Stuttgart

Das Science Café lässt sich grundsätzlich als verständigungs- und prozessorientiertes Kommunikationsverfahren bezeichnen. Form und Ausgestaltung von konkreten Veranstaltungen des Science Cafés können in der Praxis unterschiedlich sein. Ziel jeder Veranstaltung ist die Förderung des Austausches zwischen der Bevölkerung bzw. bestimmten Zielgruppen wie Jugendlichen mit Wissenschaftlern (daher die Bezeichnung „Science“ Café). Die Vorstellung, dass z. B. teilnehmende Bürgerinnen und Bürger in der Veranstaltung wie in einem Kaffeehaus sitzen und mit Experten sprechen, gibt durchaus den richtigen Eindruck von der lockeren, angenehmen und kreativen Veranstaltungsatmosphäre eines gelungenen Science Cafés wieder. Über den interaktiven Austausch sollen wissenschaftliche Themen an die Nicht-Wissenschaftler vermittelt werden. Der Austausch lohnt sich für beide Seiten, wenn er den Blick über den eigenen Tellerrand hinaus erlaubt. So finden es viele Wissenschaftler zunächst ungewohnt und manchmal herausfordernd, aber gleichzeitig spannend und gewinnbringend, ihr Forschungsthema vor Nicht-Angehörigen ihrer Disziplin darzustellen und Gedanken darüber auszutauschen. Die Gesprächszeit von zwei bis drei Stunden reicht aus, die zentralen Entwicklungslinien der wissenschaftlichen Disziplin oder des Themas zu skizzieren und dazu zentrale Reaktionen und Argumente des Publikums zu erfahren. Gerade diese Rückmeldungen sind für Wissenschaftler interessant, insbesondere wenn neue wissenschaftliche Forschungsfelder vorgestellt werden, bei denen noch unklar ist, wie Nicht-Wissenschaftler sich positionieren. Ein Erfolgsfaktor ist, das Gespräch für alle sachlichen Meinungen offen zu halten. Mit der Bildung und Erfahrung eines an wissenschaftlichen Themen interessierten Publikums stellen auch Laien „die richtigen Fragen“ und bringen relevante Perspektiven ein, was schon die zentrale Grundlage für ein spannendes Gespräch liefert.

Zum sog. Junior Science Café liegen schon detaillierte Forschungsergebnisse vor (Goldschmidt et al. 2012). Zudem existieren Leitfäden zur praktischen Umsetzung (Wissenschaft im Dialog 2011). Schülerinnen und Schüler bereiten hier meist eine Reihe von Café-Veranstaltungen vor und führen dann die Veranstaltungen selbst durch. Anfangs noch aktiv durch z. B. Lehrer betreut, übernehmen die Schülerinnen und Schüler zunehmend die Verantwortung. Dies schafft zusätzliche Lernerfolge und wertvolle Erfahrungen (,soft skills").

Fallstricke sind, dass ein Science Café wissenschaftlich interessierte Personen und weniger die „,normale Bevölkerung“ anzieht und teilweise trotzdem noch einiges an Engagement erforderlich ist, Teilnehmende zu gewinnen. Neben organisatorischen Voraussetzungen wie der Sicherung eines Veranstaltungsortes, sind die Moderation und die Vorbereitung der Experten (Rolle als offener Gesprächspartner, zielgruppengerechte Sprache) wichtige Faktoren.
\end{abstract}

Auf verschiedenen Dialogveranstaltungen (Kasten 4) stellte acatech diese Technikzukünfte interessierten Laien, Studierenden sowie Schülern vor und diskutierte sie mit ihnen. Die Formate reichten von Science Cafés über ein Seminar bis hin zum Comic-Workshop, bei dem die Teilnehmer ihre Vorstellungen in Zeichnungen visualisierten (Kasten 5, S. 55).

Die Projektgruppe lernte die Ideen und Kritikpunkte der Teilnehmer kennen und erfuhr, welche Aspekte der Künstlichen Fotosynthese sie als Chancen und welche als Risiken wahrnehmen. Viele befürchteten, dass die gentechnisch veränderten Organismen freigesetzt werden könnten, etwa bei Unfällen. Kritische Fragen betrafen den Wirkungsgrad und die Wirtschaftlichkeit. Auch der Wasser- und Energieverbrauch sowie der Einsatz von Dünger wurden skeptisch betrachtet. Als Chance bewerteten die Teilnehmer die Verwendung von Industrieabgasen.

Der Ansatz der Technikzukünfte hat sich in den Dialogformaten bewährt. Die Geschichten eröffneten den Teilnehmern einen Zugang zur Künstlichen Fotosynthese, machten die Technologie für Laien verständlich und dienten als Ausgangspunkt für Diskussionen.

\section{Ausblick}

Biotechnologie ist ein gut geeignetes Studienobjekt der Wissenschafts- und Technikkommunikation: Ihre Auswirkungen (zumal in Medizin und Ernährung) und die damit verbundenen Kontroversen, die von moralischen Aspekten, wie die Stellung des Menschen in seiner Umwelt über den Verbraucherschutz bis hin zu Gesundheitsfragen und Umweltauswirkungen reichen, sind denkbar vielfältig. Das Thema betrifft auf die eine oder andere Weise jeden. Die jahrzehntelange Diskussion um den Einsatz der Gentechnik in Medizin und Landwirtschaft sind ausführlich dokumentiert und analysiert worden.

Welche Konsequenzen daraus für eine zukünftige Biotechnologie-Kommunikation gezogen werden, ist freilich kein Glasperlenspiel. Überspringen wir hier einmal die Diskussionen um die Synthetische Biologie (DFG et al. 2009), geht es jetzt um eine genetische Methode, mit der das Genom ,so einfach und schnell wie noch nie“ zuvor (Knox 2015, S. 22) verändert werden kann. Das Verfahren hinterlässt keine Spuren, die Produkte können mithin nicht unterschieden werden von natürlichen Mutationen. Die Schlagzeilen lauten (wieder einmal) „Menschen nach 
Kasten 5: Comic von Philipp Schrögel
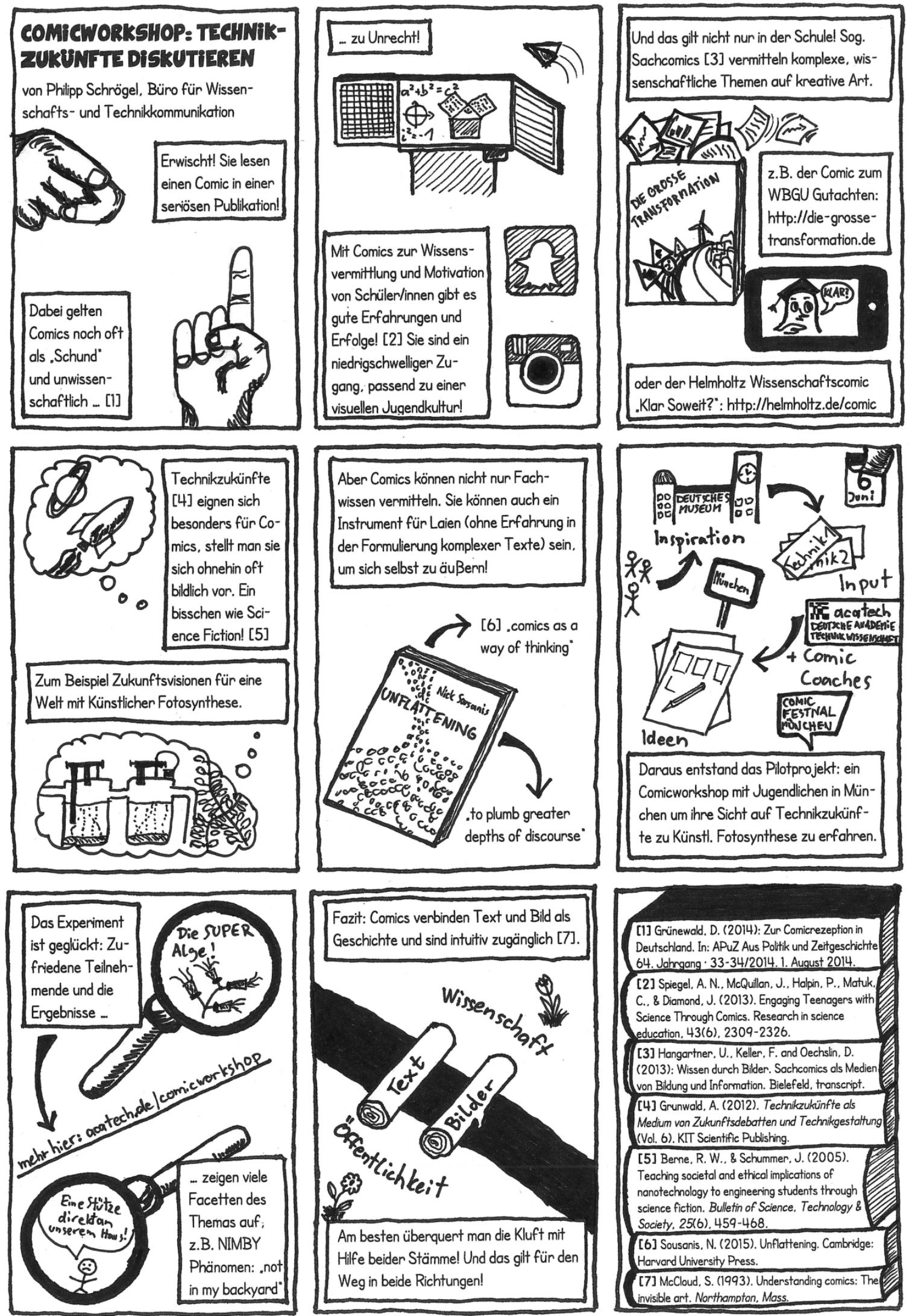
Maß“, „Im Reich der neuen Tiere“ und „Das Ende des Lebens, wie wir es kennen".

Chinesische Forscher haben mit CRISPR/ $\mathrm{Cas}^{8}$ tatsächlich in das Erbgut menschlicher Embryonen eingegriffen. Ziel wäre es, ein Gen zu verändern, das in mutierter Form eine schwere Krankheit verursachen kann. Und CRISPR/Cas sowie anderen Verfahren der sog. Genom-Chirurgie sind keineswegs auf medizinische Anwendungen „beschränkt“: Resistente Unkräuter sollen anfällig gemacht werden für Herbizide, ausgestorbene Tierarten sollen wieder zum Leben erweckt werden. Eher konkret ist die Idee, Moskitomücken auszurotten, indem das Erbgut einzelner Insekten verändert wird: Die schädlichen Mutationen sollen sich dann auf alle Nachkommen kopieren und sich damit innerhalb weniger Generationen in der gesamten Population ausbreiten.

Wenn es eine Lehre aus der Technikentwicklung der vergangenen Jahrhunderte gibt, dann die, dass große Versprechungen von unintendierten Nebenfolgen begleitet werden. So empfehlen die Wissenschaftsakademien, neben dem innerwissenschaftlichen Dialog ,die Debatte zu wissenschaftlichen, ethischen und rechtlichen Möglichkeiten, Grenzen und Konsequenzen des genome editing in die Öffentlichkeit“ zu tragen, ,,insbesondere mit Blick auf therapeutische Anwendungen und gezielte, potenziell weitreichende Eingriffe in Ökosysteme." (Leopoldina et al. 2015)

Noch deutlicher und konkreter wird der Wissenschaftsforscher Daniel Sarewitz in einem Kommentar in Nature (Sarewitz 2015): Nicht nur eine breite Diskussion tut not, sondern auch die Entscheidung über den Einsatz muss gemeinsam gefunden werden. Falls es in einer demokratischen Gesellschaft nicht gelingt, Fragen zur GenomChirurgie gemeinsam zu entscheiden, also auch mit den Gruppen außerhalb der Wissenschaft, bekommt Wissenschaft ein Problem: ,The idea that the risks, benefits and ethical challenges of these emerging technologies are something to be decided by experts is wrong-headed, futile and self-defeating. It misunderstands the role of science in public discussions about technological risk. It seriously underestimates the democratic sources of science's vitality and the capacities of democratic deliberation. And it will further delegitimize and politicize science in modern societies."
Immerhin: Viele Erfahrungen und ein breites Instrumentarium der Biotechnologie-Kommunikation stehen bereit.

\section{Anmerkungen}

1) http://www.faz.net/aktuell/wirtschaft/verlagerung-nach-amerika-basf-gibt-gruene-gentechnikin-europa-auf-11608862.html

2) http://www.pflanzen-forschung-ethik.de/

3) $\mathrm{http}: / /$ www.biotechnologie.de/

4) http://www.keine-gentechnik.de/

5) http://www.senseaboutscience.org/

6) http://www.sciencemediacentre.org/

7) Mittlerweile ist in Deutschland ebenfalls ein SMC entstanden, s. https://www.sciencemediacenter.de/

8) Clustered Regularly Interspaced Short Palindromic Repeats (CRISPR) ist eine biochemische Methode, um DNA gezielt zu schneiden und zu verändern.

\section{Literatur}

acatech-Deutsche Akademie der Technikwissenschaften, 2011: Akzeptanz von Technik und Infrastrukturen. Anmerkungen zu einem aktuellen Gesellschaftlichen Problem (acatech Position). Heidelberg

acatech - Deutsche Akademie der Technikwissenschaften, 2012a: Perspektiven der BiotechnologieKommunikation. Kontroversen - Randbedingungen - Formate (acatech Position). Berlin

acatech - Deutsche Akademie der Technikwissenschaften, 2012b: Technikzukünfte. Vorausdenken Erstellen - Bewerten (acatech Impuls). Heidelberg

acatech - Deutsche Akademie der Technikwissenschaften, 2016: Technik gemeinsam gestalten (acatech Impuls)

BMBF - Bundesministerium für Bildung und Forschung, 2014: Die neue Hightech-Strategie Innovationen für Deutschland; http://www.bmbf.de/pub_hts/ HTS_Broschure_Web.pdf (download 28.01.2016)

BUND - Bund für Umwelt und Naturschutz Deutschland e.V., 2012: Nachhaltige Wissenschaft. Plädoyer für eine Wissenschaft für und mit der Gesellschaft. (Diskussionspapier); http://www.bund.net/fileadmin/ bundnet/publikationen/nachhaltigkeit/20110202_ nachhaltigkeit_wissenschaft_diskussion.pdf (download 28.1.16)

DFG - Deutsche Forschungsgemeinschaft; acatech - Deutsche Akademie der Technikwissenschaften; Deutsche Akademie der Naturforscher Leopoldina, 
2009: Synthetische Biologie (Synthetic Biology). Stellungnahme (Position). Weinheim

EC-European Commission, 2013: Science and Society. Time for a New Deal. http://ec.europa.eu/archives/commission_2010-2014/president/advisory-council/documents/berl_papers_issue_3.pdf(download 28.1.16)

Goldschmidt R.; Scheel, O.; Renn, O., 2012: Zur Wirkung und Effektivität von Dialog- und Beteiligungsformaten. In: Stuttgarter Beiträge zur Risiko- und Nachhaltigkeitsforschung 23 (2012)

Kahan, D.; Braman, D.; Slovic, P. et al., 2009: Cultural Cognition of the Risks and Benefits of Nanotechnology. In: Nature Nanotechnology 4 (2009), S. 87-90

Knox, M., 2015: Gezielter Eingriff ins Erbgut. In: Spektrum der Wissenschaft (2015), S. 22

Nationale Akademie der Wissenschaften Leopoldina; DFG - Deutsche Forschungsgemeinschaft; acatech - Deutsche Akademie der Technikwissenschaften; Union der deutschen Akademien der Wissenschaften, 2015: Chancen und Grenzen des Genome Editing (The Opportunities and Limits of Genome Editing). Halle (Saale)

Nowotny H.; Scott P.; Gibbons M., 2001: Rethinking Science. Knowledge in an Age of Uncertainty. Cambridge

Sarewitz, D., 2015: CRISPR: Science Can't Solve it. In: Nature 522/7557 (2015), S. 413-414

Schneidewind, U., 2013: Transformative Literacy. Gesellschaftliche Veränderungsprozesse verstehen und gestalten. In: GAIA 22/2 (2013), S. 82-86

Weitze, M.-D.; Pühler, A.; Heckl, W.M. et al. (Hg.), 2012: Biotechnologie-Kommunikation. Kontroversen, Analysen, Aktivitäten (acatech Diskussion). Berlin

Wissenschaft im Dialog, 2011: Leitfaden Junior Science Café. Schüler plaudern über Wissenschaft. Berlin

\section{Kontakt}

Dr. Marc-Denis Weitze

acatech - Deutsche Akademie der

Technikwissenschaften

Karolinenplatz 4, 80333 München

Tel.: +49 (0)89 / 5203 09-50

E-Mail: weitze@acatech.de

\section{Autorenhinweise}

Wir bitten alle Autorinnen und Autoren, die ein Manuskript bei TATuP einreichen, die folgenden Hinweise zu beachten:

Umfang: Eine Druckseite umfasst max. 3.500 Zeichen (ohne Leerzeichen). Für den Umfang eines Beitrags ist die Rubrik, in der er erscheint, ausschlaggebend. Genauere Angaben erhalten die Autoren von der Redaktion.

Abstract: Autoren, deren Beiträge im Themenschwerpunkt des Heftes oder in den Rubriken TA-Konzepte und -Methoden und Diskussionsforum sowie TA-Projekte erscheinen, werden gebeten, ihrem Beitrag ein Abstract voranzustellen, in dem eine kurze inhaltliche Übersicht über den Beitrag gegeben wird. Die Länge dieses Abstracts sollte 780 Zeichen (ohne Leerzeichen) nicht überschreiten.

Abbildungen, Diagramme und Tabellen: Abbildungen und Tabellen sind sowohl in das eingereichte Manuskript einzufügen sowie auch getrennt von der ersten Fassung des Manuskripts einzusenden. Abbildungen und Tabellen bitte mit Überschrift und Quellenangabe versehen. Wurden sie vom Autor selbst erstellt, bitte die Formulierung ,eigene Darstellung“ als Quellenangabe verwenden Zum Format: Tabellen sind als Word-Datei, Diagramme in Excel und Abbildungen in Adobe Illustrator oder Powerpoint zu liefern. Sollten Sie lediglich andere Formate zur Verfügung haben, wenden Sie sich bitte frühzeitig an die Redaktion. Aus Gründen der Seitenplanung und des Layouts liegt die Entscheidung über die endgültige Größe und Platzierung der Abbildungen und Tabellen innerhalb des Beitrags bei der Redaktion.

Bibliografische Angaben: Die zitierte Literatur wird am Ende des Beitrags als Liste in alphabetischer Reihenfolge angegeben. Im Text selbst geschieht dies in runden Klammern (z. B. Wiegerling 2011); bei Zitaten ist die Seitenangabe hinzuzufügen (z. B. Fink/Weyer 2011, S. 91). Bei den Angaben in der Literaturliste orientieren Sie sich bitte an folgenden Beispielen:

Monografien: Wiegerling, K., 2011: Philosophie intelligenter Welten. München

Bei Aufsätzen: Fink, R.D.; Weyer, J., 2011: Autonome Technik als Herausforderung der soziologischen Handlungstheorie. In: Zeitschrift für Soziologie 40/2 (2011), S. 91-111

Bei Beiträgen in Sammelbänden: Mehler, A., 2010: Artifizielle Interaktivität. Eine semiotische Betrachtung. In: Sutter, T.; Mehler, A. (Hg.): Medienwandel als Wandel von Interaktionsformen. Heidelberg

Bei Internet-Quellen: Waterfield, J., 2006: From Corporation to Transnational Pluralism. London; http://www.plugin-tot.com (download 12.3.09) 